\title{
Lupus Biomarker Discovery, Validation, Approval, and Impact on Clinical Trials
}

Joseph M. Ahearn, MD*

Chau-Ching Liu, MD, PhD

Susan Manzi, MD, MPH

\author{
Address \\ *Lupus Center of Excellence, Autoimmunity Institute, Allegheny Health Network, \\ 320 E. North Avenue, Pittsburgh, PA 15212, USA \\ Email: jahearn@wpahs.org
}

Published online: 11 January 2015

(C) Springer International Publishing AG 2015

This article is part of the Topical Collection on Lupus

Keywords Lupus · Biomarkers · Validation · FDA · LDT · Lupology

\section{Opinion statement}

Current estimates suggest that bringing a new therapeutic from the laboratory to the patient requires 16-17 years and US\$4-5 billion. One of the challenges in lupus clinical trials is the dearth of available biomarkers to identify those most likely to respond, to demonstrate response, and to predict adverse events. The lupus biomarker discovery pipeline, much like the lupus pharmaceutical discovery pipeline, has seen many potential advances but enjoyed all too few ultimate successes. New lupus biomarkers are essential and certain to accelerate lupus drug discovery and to move "lupology" from being less of an art to being more of a science. There is no precise path to lupus biomarker validation, FDA approval is generally not required, a multidisciplinary team is essential to optimize success, and a commercial partner is almost required to move advances in academia through the translational process. Despite the relatively few successes to date, the trajectory for lupus biomarker discovery and validation is steep and more promising than ever. Tandem and synergic advances in lupus biomarker validation and approval of new lupus therapeutics are hopefully on the horizon.

\section{Introduction}

Whereas the dearth of FDA approved drugs for lupus during the past half century is often cited by physicians, patients, investigators, and lupus advocacy groups, the parallel slow pace in the discovery of clinically useful lupus biomarkers tends to be recognized as an important but less pressing issue. The following will focus on 
describing the current state of lupus biomarker discovery and suggest that accelerating the lupus biomarker discovery pipeline is essential to accelerating the lupus drug discovery process.

\section{What is a lupus biomarker?}

A lupus biomarker can be defined as any measurement that accurately and reproducibly indicates some aspect of the lupus disease process. This definition is adapted from the National Institutes of Health Biomarkers Definitions Working Group publication in 1998 that is most commonly referred to in the general field of biomarker discovery and validation [1]. Categories of lupus biomarkers include those that can be used to predict susceptibility, diagnose the disease, monitor disease activity, predict or indicate a disease flare, predict or indicate response to a specific therapeutic intervention, stratify, or subset patients who have or are at increased risk of having involvement of a specific organ system such as brain, heart, or kidney, and those biomarkers that measure some aspect of organ damage. Although this list is not comprehensive, it includes the majority of lupus biomarkers that are currently in use, under investigation, or being considered in lupus discovery efforts.

\section{What are the current lupus biomarkers?}

A comprehensive review of specific lupus biomarkers that are currently in the discovery and validation process has recently been published elsewhere [2]. Lupus biomarkers can be grouped into several categories that are not necessarily mutually exclusive. First, there are hundreds of reports of various measurements performed in patients with lupus that have identified biomarkers that "correlate with," "associate with," "reflect," "predict," or otherwise relate to some aspect of the lupus disease process. The vast majority of these reports are single center cross-sectional pilot studies, and this is the appropriate starting point for almost any lupus biomarker discovery effort. The second category of lupus biomarkers consists of those that have successfully advanced from the single center crosssectional pilot study to a single center study that confirms with a "test" data set what the pilot "training" data set demonstrated in the initial exploratory investigation. The third category contains those biomarkers that have been validated by multicenter study, a process that will be further discussed below. Finally, there are those lupus biomarkers that are currently in clinical use by practicing physicians, investigators, and those conducting lupus clinical trials.

Largely due to historical reasons, lupus biomarkers in current use have not necessarily moved through the pilot trial, single center test, and multicenter validation process. As noted above, the concept and definition of biomarkers in general were nascent circa 2001 and the lupus biomarker field is still in its infancy. That being said, lupus biomarkers in current use are either those that are highly specific to lupus as well as those that are used in the management of other diseases. Some of the lupus-specific biomarkers include measurements of anti-dsDNA, anti-Sm, and serum C3 and C4 as well as the numerous indices that have been developed primarily for research efforts such as the Systemic Lupus Activity Measure (SLAM), the University of Toronto SLE Disease Activity 
Index (SLEDAI), the SELENA-SLEDAI, and the British Isles Lupus Assessment Group (BILAG) scale [3, 4]. Lupus biomarkers in current use that are not specific to the disease include measurements such as proteinuria, ESR, CRP, CBC, ANA, anti-Ro/SSA anti-La/SSB, EKG, CXR, and similar laboratory studies fundamental to the practice of medicine.

\section{What are the characteristics of an ideal lupus biomarker?}

The value in lupus biomarkers is that they are objective signs of the disease in contrast to subjective symptoms. The lupus biomarker discovery and validation effort can therefore be considered a path to transforming the practice of lupus patient care from less of an art to more of a science. For example, neuropsychiatric systemic lupus erythematosus (NPSLE) is arguably the most common yet least understood manifestation of the disease. Patients who report subjective symptoms such as headache, memory loss, mood disturbances, and other of the myriad manifestations of NPSLE pose an enormous challenge to the treating physician. In contrast to cutaneous eruptions that may be visible and obvious, the lupus brain cannot be immediately visualized, biopsied, or otherwise examined in the provider's office. Thus, current efforts to develop novel methods of imaging and identifying molecules in the cerebrospinal fluid, blood, plasma, or serum are critical to improving the care of patients with lupus and further transforming "lupology" from less of an art to more of a science [5]. This would enhance lupus patient care not only in the hands of the "lupologist" but also as delivered by the general rheumatologist and by the primary care physician.

The ideal lupus biomarker is one that can be used as a surrogate marker or surrogate endpoint for a clinical endpoint. A clinical endpoint measures how a patient feels, functions, or survives and as such reflects morbidity and mortality in a specific disease. Lupus clinical endpoints can be either objective (e.g., death, stroke, myocardial infarction, renal transplant) or subjective (e.g., quality of life and other patient reported outcomes (PRO)), and they are the determinants by which new drugs for lupus are approved. Surrogate endpoints are essential for lupus patient care and drug discovery because clinical endpoints may be potentially unreliable (subjective), rare (e.g., death), invasive (renal biopsy), or otherwise inconvenient, unethical, cumbersome, or suboptimal for use. A lupus surrogate endpoint is a substitute for a clinical endpoint. Examples in other fields include hemoglobin A1c in diabetes, CD4 counts in HIV/AIDS, hypertension and cholesterol in cardiovascular disease, and TSH in thyroid disorders.

Several characteristics of a lupus biomarker are essential if it is to be validated and adopted as a surrogate marker. The measurement should be sensitive, specific, precise, accurate, stable, and cost-effective if it is to be effective and widely adopted in patient care and/or drug discovery.

\section{What is lupus biomarker validation?}

There is no precise path to validation of biomarkers for lupus or for any other disease. This has led to misunderstanding, confusion, and perhaps delays in lupus biomarker validation. Validation of a lupus biomarker should be both technical/analytical and clinical. In essence, a validated lupus biomarker is one 
that successfully passes through rigorous peer review, is recommended by key opinion leaders, and has been demonstrated to influence clinical decision making. As such, the context for us of the lupus biomarker must be considered and the validation process will likely vary accordingly. One recent example of lupus biomarker validation is the Avise SLE diagnostic test, which is based upon the cell-bound complement activation products (CB-CAPs) technology platform [6]. The Avise discovery and validation process began with a single center pilot and confirmation studies, followed by a multicenter trial at 14 sites, each of which was directed by an investigator with lupus expertise.

In addition to validating that the lupus biomarker is important for clinical decision(s), a lupus biomarker should also undergo analytical/technical validation. This ensures that the biomarker test is measuring what it is intended to measure, that performance of the assay is within acceptable inter-operator and intra-operator variations, and that the influence of time and temperature factors such as freeze-thawing, shipping at ambient temperatures or on ice, and delay from obtaining a sample to performing the assay whether in minutes, hours, or days. This last issue has been generally overlooked in the biomarker discovery field, but it is now receiving the long overdue attention that it deserves and is actually recognized as a distinct discipline referred to as biosampling or biospecimens science which is focused on sample fixation, storage, transport, and other influences to which it is subjected from procurement to assay result. These challenges faced in biomarker validation are the specific focus of Biopreservation and Biobanking, the official journal of the International Society of Biological and Environmental Repositories.

Remarkably, many of the lupus biomarker assays in current use have never undergone rigorous analytical validation and as such there are no standard of care validated protocols for tests such as ANA and anti-dsDNA although the results of these assays significantly influence clinical decision making. This is not unusual in the general field of commercialized biomarkers. For example, other tests developed decades ago such as prostate-specific antigen (PSA) and alpha-fetoprotein (AFP) were never formally validated or prospectively studied [7]. Biomarker validation will now likely be required for all future potential lupus biomarkers discovered in the laboratory to reach the treating physician and patient.

\section{What is the role of the FDA in lupus biomarker adoption?}

Validation, commercialization, adoption, and clinical use of most lupus biomarkers do not currently require FDA approval. The main exception to this would be a companion lupus biomarker that guides prescription of a therapeutic and would be indicated as such on the label. Those engaged in the biomarker development pipeline may actually be reluctant to seek FDA approval for their test for several reasons. First, the process is time consuming, expensive, high risk, and, according to some, can be arbitrary. In the end, a low return on investment of both capital and sweat equity may be realized. Second, pursuit of FDA approval of a biomarker may result in rejection not because the test is inaccurate but due to other factors that may not be directly related to product performance such as lack of understanding of the field by reviewers, low priority, personal agendas, etc. At least one perspective from a seasoned 
investigator, innovator, entrepreneur, and investor presents a decidedly unfavorable view of the FDA and potential bureaucratic nightmares that might confront a highly qualified sponsor seeking approval for a medical device. Innovation Breakdown [8], published by Joseph Gulfo, MD, MBA in 2014, chronicles a decade-long experience in seeking FDA approval for a device developed to aid dermatologists in early detection of melanoma. Although this is the account of an individual, it does present a passionate case for changes designed to help everyone in the medical device (substitute biomarker) pipeline including innovators, investors, physicians, and most importantly, patients. A third source of reluctance for seeking FDA approval of biomarkers is that intellectual property is not protected in the process. The time-consuming effort can be unpredictably delayed with "time outs," and no guarantee that the shelf life of the patent(s) will be extended accordingly. Fourth, the FDA may request longitudinal studies that require further time and cost without protection from competing companies with the capacity to "design around" the invention for which approval is being sought. Fifth, FDA approval is no guarantee of commercial success, i.e., market adoption by physicians and patients. A product developed by a company seeking FDA approval can be dominated by a competitive product that is not approved but has enjoyed a more successful marketing campaign. Sixth, FDA approval is no guarantee that a test will be reimbursed by payers. There are many examples of medical devices, tests, and procedures available to patients that have been FDA approved but are not reimbursed, adding further economic burden to the biomarker discovery and validation process.

As a more rapid, economical, predictable, and controllable path to biomarker adoption, some companies have taken the alternative path to establish a laboratory-developed test (LDT). An LDT also referred to as an "in-house" or "home brew" test is one that has been designed and manufactured and is used in a single laboratory. LDTs are accepted as being scientifically valid and are in widespread use in delivery of health care in the USA. LDTs do not require FDA approval but rather are accredited through the Clinical Laboratory Improvement Amendments (CLIA) as administered by the Centers for Medicaid and Medicare Services (CMS) and by the American College of Pathology (ACP). Such accreditation values accuracy, precision, reproducibility, analytic sensitivity, analytic specificity, reference range, and other measures of quality control. LDTs are considered high complexity tests, are not generally subject to FDA review, and are considered "devices." Laboratories that perform LDTs are carefully and continually monitored. The LDT process, in contrast to the FDA approval process, fosters rapid innovation, incremental advances, timely return on investment, and a potentially less capricious path to moving a lupus biomarker from the bench to the bedside.

\section{How do lupus biomarkers impact drug discovery?}

Current estimates suggest that the time required for a laboratory discovery to be translated successfully into a new prescription for a patient is 16-17 years at a cost of US $\$ 4-5$ billion, and one of the most significant bottlenecks in the process is lack of informative disease biomarkers [9]. The overall critical role of biomarkers in drug discovery has been thoroughly reviewed recently [10]. The 
dearth of validated lupus biomarkers that can serve as surrogate endpoints in clinical trials is undoubtedly a culprit in the widespread failure of lupus clinical trials to date.

Lupus biomarkers that have been demonstrated to be surrogate endpoints can play several potential roles in the drug discovery pipeline. First, lupus biomarkers can increase the diagnostic accuracy of patients who are enrolled in a clinical trial. Misdiagnosis of lupus is well recognized. In one report, $47 \%$ of patients referred to a lupus clinic were misdiagnosed by referring primary care physicians and $27 \%$ of those referred by a rheumatologist were misdiagnosed incorrectly as having lupus [11]. In the context of recruitment for multicenter national or international clinical trials for a potential lupus therapeutic, it is conceivable that patients enrolled with a diagnosis of "lupus" may be misdiagnosed and therefore contaminate the study. A more accurate objective lupus diagnostic biomarker might improve the chances of a successful lupus clinical trial. Second, lupus biomarkers might assist in the identification of patients more likely to respond to a potential therapeutic. For example, if a novel biologic therapy was targeted at the interferon pathway, the drug might be more likely to be effective in those lupus patients demonstrated to have an abnormal interferon signature $[12,13]$. Surrogate endpoints may be most useful if they are actually components of the pathophysiologic pathway being targeted by the intervention being evaluated. The capacity of a clinical trial to focus on patients most likely to be responders and avoid dilution with those who are least likely to respond will obviously increase the chances of successfully reaching the trial endpoints. Third, lupus biomarkers can increase the chances of success in a clinical trial by serving as surrogate endpoints for an intervention intended to elicit a response. Fourth, lupus biomarkers can be useful in monitoring drug toxicity or identifying patients at increased risk for toxicity in a clinical trial.

Perhaps, the best known recent example of a successful companion biomarker is the Her-2-Herceptin story. Since patients with breast cancers that overexpress Her-2 are most likely to respond to Herceptin, once the biomarker was validated, it was possible to significantly decrease the number of patients required for study and duration of follow-up in the trial from 2200 to 470 subjects and from $~ 10$ to 1.6 years, respectively [14].

Just as lupus biomarkers can inform clinical trials, it should be recognized that clinical trials have great potential to inform the lupus biomarker discovery and validation process. This is likely to be accomplished through examination and comparison of responders versus non-responders, those who experience adverse events versus those who do not, etc. with genomic, proteomic, candidate biomarker, and similar types of studies.

\section{Conclusion: future directions for lupus biomarkers}

The lupus biomarker discovery and validation process is inherently difficult. However, the challenges in this process are not unique to lupus as reflected in a recently published study that documented less than 100 validated biomarkers in clinical use among more than 150,000 published biomarker claims, a success rate of 1 per 1500 [15]! The 
following ten points are some of those that should be considered while undertaking lupus biomarker discovery and validation.

1. Every lupus biomarker discovery effort begins by asking a question, and it is essential to ask the right question and design the scientific approach accordingly. For example, there is a distinct but underappreciated difference in the path for discovering a biomarker to diagnose early lupus versus a biomarker to diagnose lupus earlier.

2. Biomarker discovery and validation efforts may require extremely broad multidisciplinary teams including not only physicians and scientists but also those with expertise, in biostatistics, biopreservation and biobanking, bioethics, business and entrepreneurship, regulatory guidelines and restrictions, legal, and beyond.

3. Failures in biosampling and biobanking are now recognized as a core problem in biomarker research and the basis for generally nonreproducible results in the field. The potential influence of time, temperature, fixation, shipping, freezing, and other factors should be considered and tested during the validation process.

4. Lupus biomarker studies should be on a path to validation that compares potential new biomarkers to the gold standards that are in clinical practice, even if those that have been adopted were never validated. For example, a potential urinary protein biomarker that is demonstrated to be elevated in lupus nephritis must be compared with proteinuria and demonstrated to hold greater or at least equivalent value.

5. Validated incremental advances should be embraced and adopted. The validation process does not have a defined endpoint. As new data are generated, many technical and clinical aspects of the biomarker will be refined such that the process should not become paralyzed by perfection at any specific point.

6. Translating a biomarker from the laboratory to the bedside is a time consuming and expensive process that almost certainly requires a commercial partner. Resources from academia and funding agencies ultimately expire at some point in the pipeline.

7. It is essential to recognize the enormous value inherent in lupus biomarker panels, which is undoubtedly where the field is headed. For example, five diagnostic biomarkers with $100 \%$ sensitivity and $20 \%$ specificity individually have the potential to be a perfect diagnostic panel if each is specific for a distinct, non-overlapping subset of patients.

8. Animal models are a potential source to be mined for lupus biomarkers that will translate and impact human lupus patient care and drug development. However, too often such potential biomarkers in other species such as the mouse are pursued without adequate consideration of homology, analogy, and true potential for translation. The potential value in mouse to human translation in light of the disconnect between mouse and human lupus has recently been carefully analyzed by comparing four different mouse models of lupus for their respective 
capacities to inform human lupus biomarker validation and clinical trials [16].

9. Reimbursement policies for biomarkers, particularly for those that are surrogate endpoints must be reevaluated. Whereas the time and cost for bringing a new drug to market is well recognized, the critical role of biomarkers in this process is not. Commercial partners are critical to the lupus biomarker pipeline but they will be reluctant to engage in the process if the likelihood of reimbursement is not recognized.

10. Lupus biomarker discovery efforts to date have been generally disappointing; however, the current pace of discovery in the field is on a steep trajectory. Failures in previous efforts have been highly informative, and the outlook for success in the near future is brighter than ever.

\section{Compliance with Ethics Guidelines}

\section{Conflict of Interest}

Joseph M. Ahearn reports personal fees from Exagen Diagnostics, outside the submitted work. In addition, Dr. Ahearn is an inventor of patents related to Cell-Bound Complement Activation Products for Lupus Diagnosis and Monitoring, owned by University of Pittsburgh with exclusive license held by Exagen Diagnostics.

Chau-Ching Liu reports a research grant from Exagen Diagnostics, outside the submitted work. In addition, Dr. Liu is inventor of patents related to Cell-Bound Complement Activation Products for Lupus Diagnosis and Monitoring, owned by University of Pittsburgh with exclusive license held by Exagen Diagnostics.

Susan Manzi reports personal fees from Exagen Diagnostics, outside the submitted work. In addition, Dr. Manzi is inventor of patents related to Cell-Bound Complement Activation Products for Lupus Diagnosis and Monitoring, owned by University of Pittsburgh with exclusive license held by Exagen Diagnostics.

\section{Human and Animal Rights and Informed Consent}

This article does not contain any studies with human or animal subjects performed by any of the authors.

\section{References and Recommended Reading}

1. Biomarkers Definitions Working Group. Clin Pharmacol Therapeut. 2001;69:89-95.

2. Ahearn JM, Chau-Ching L, Manzi S. Biomarkers for systemic lupus erythematosus. Transl Res. 2012;159:326-42.

3. Liang MH, Socher SA, Larson MG, Schur P. Reliability and validity of six systems for the clinical assessment of disease activity in systemic lupus erythematosus. Arthritis Rheumatism. 1989;32:1107-18.

4. Petri M, Kim MY, Kalunian KC, Grossman J, Hahn B, Sammaritano LR, et al. For the OC-SELENA trial. Combined oral contraceptives in women with systemic lupus erythematosus. N Engl J Med. 2005;353:2550-8.

5. DeGiorgio LA, Konstantinov KN, Lee SC, et al. A subset of lupus anti-DNA antibodies cross-reacts with the
NR2 glutamate receptor in systemic lupus erythematosus. Nat Med. 2001;7:1189-93.

6. Ahearn JM, Manzi S. The lupus biomarker odyssey: one experience. Methods Mol Biol. 2014;1134:17-35.

7. Amur S, Frueh FW, Lesko LJ, Huang S-M. Integration and use of biomarkers in drug development, regulation and clinical practice: a US regulatory perspective. Biomarkers Med. 2008;2:305-11.

8. Gulfo, J. Innovation Breakdown: How the FDA and Wall Street Cripple Medical Advances, Post Hill Press, 2014.

9. Staff NP, Runge BK, Windebank AJ. Breaking down translation barriers: investigator's perspective. Sci Transl Med. 2014;6:1-5. 
10. Anderson DC, Kodukula K. Biomarkers in pharmacology and drug discovery. Biochemicsl Pharmacol. 2014;87:172-88.

11. Narain S, Richards H, Satoh M, Sarmiento M, Davidson $\mathrm{R}$, Shuster J, et al. Diagnostic accuracy for lupus and other systemic autoimmune diseases in the community setting. Arch Intern Med. 2004;164:2435-41.

12. Crow MK, Kirou KA. Interferon-alpha in systemic lupus erythematosus. Curr Opin Rheumatol. 2004;16:541-7.

13. Feng $\mathrm{X}, \mathrm{Wu} \mathrm{H}$, Grossman JM, et al. Association of increased interferon-inducible gene expression with disease activity and lupus nephritis in patients with systemic lupus erythematosus. Arthritis Rheumatism. 2006;54(9):2951-62.

14. Zwierzina H. Biomarkers in drug development. Ann Oncol. 2008;19(S5):v33-7.

15. Poste G. Bring on the biomarkers. Nature. 2011;469:156-7.

16. Bender AT, Wu Y, Cao Q, Ding Y, Oestreicher J, Genest $\mathrm{M}$, et al. Assessment of the translational value of mouse lupus models using clinically relevant biomarkers. Transl Res. 2014;163:515-32. 